\title{
Alumnos de empresa vs alumnos por cuenta propia en la formación online. Análisis de su desarrollo y éxito aplicado a un curso a distancia en hidráulica
}

\author{
del Teso, R. ${ }^{\text {a1 }}$, Estruch-Juan, E. ${ }^{\text {a2 }}$, Gómez, E. ${ }^{\text {a3, }}$ Soriano, J. ${ }^{\text {a4 }}$ \\ Universitat Politècnica de València.

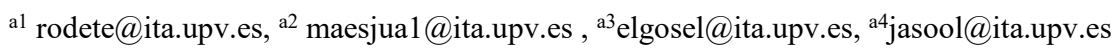

aTA, Departamento de Ingeniería Hidráulica y Medio Ambiente. Escuela Técnica Superior de Ingenieros Industriales.

\begin{abstract}
The training of active workers has been growing unstoppably in recent years. Companies are increasingly demanding qualified personnel and funding specific training for their employees. Within this framework, the demand for e-learning courses offered by the ITA can be divided into two types of students, those who register on a personal basis and those who do so through their company. The training received by both groups is identical, and they must achieve the same learning outcomes.
\end{abstract}

In this paper we analyse different variables involved in the learning process of the course with more participants than any other offered by ITA: Analysis of water networks with EPANET. The aim of the study is to detect if there is a lack of motivation on the part of the students coming from companies, and determine the most significant differences between the students of both groups. Results will point the way to readapt and improve students' learning regardless of their type.

Keywords: e-learning, online courses, employee training, employment and training, learning analysis.

\section{Resumen}

La formación de trabajadores en activo está creciendo de forma imparable en los últimos años. Las empresas cada vez demandan más personal cualificado, apostando por ofrecer o costear una formación específica para el puesto de trabajo a desempeañar por sus empleados. Dentro de este marco, la demanda de formación a distancia ofrecida por el ITA, se puede dividir en dos tipos de alumnos, los que se inscriben de forma particular, y los que lo hacen a través de su empresa. La formación que reciben ambos grupos es idéntica, debiendo alcanzar los mismos resultados de aprendizaje.

En este trabajo se analizan diferentes variables que intervienen en el proceso de aprendizaje del curso con más participantes de los ofertados por el ITA: Análisis de redes de agua con EPANET. El objetivo es detectar si existe una falta de motivación por parte de los alumnos provenientes de empresas, y de poder concluir las diferencias más significativas entre los alumnos de ambos grupos. Esto permitirá actuar de forma pertinente para readaptar y mejorar el aprendizaje de los estudiantes.

Palabras clave: formación a distancia, cursos online, formación para empresas, empleo y formación, análisis del aprendizaje. 


\section{Introducción}

El número de estudiantes matriculados en posgrados ha crecido más de un 250\% entre el curso 2008/2009 y el 2017/2018 (crue, 2018). Esta tendencia puede estar relacionada con las tasas de desempleo, puesto que cuanto mayor es la formación de una persona, menos riesgo tendrá de estar en situación de desempleo según se desprende de algunos estudios, como el realizado por el Instituto Nacional de Estadística (INE), en el cual se indida que la tasa de empleo en 2019 de los universitarios graduados en el curso 2013-2014 fue del $86,1 \%$, mientras que la de titulados en Máster en ese mismo curso asciende hasta el 87,3\%. En la rama de ingeniería y arquitectura, estas cifras ascienden hasta el $92,4 \%$ en graduados universitarios y de Máster, lo que indica que la tasa de desempleo se sitúa en un 4,6\% (INE, 2020).

Estos datos se ven reforzados por las perspectivas futuras sobre formación y empleo, ya que se estima que en 2026 los trabajadores con formación media (bachiller o formación profesional) y alta (estudios de grado o superiores) representarán el 57\% del total de trabajadores en activo, un 10\% más que en 2016 (Oliver, 2018). Además, se espera que entre 2016 y 2026 se generen 2,6 millones de empleos de los cuales 2,3 millones tendrán un nivel de formación alto. En cambio, los trabajos sin cualificación disminuirán un $0,7 \%$ anual en ese mismo periodo (Oliver, 2018).

Casi el $40 \%$ de las ofertas de trabajo que requieren un nivel de formación determinado, solicitan titulados universitarios (Infoempleo y Adecco, 2019). Las empresas buscan un perfil de empleado con formación superior, pero además, muchas de ellas apuestan por que sus empleados sigan formándose en aspectos específicos del puesto de trabajo que desarrollan o van a desarrollar.

Según se desprende del informe "Formación para el empleo. Balance de situación 2019", desarrollado por la Fundación Estatal para la Formación en el Empleo (FUNDAE), en 2019, el presupuesto global de formación dirigido a trabajadores ocupados en España fue de 1.147 millones de euros. El 58\% de estos fondos se destinó a bonificaciones para empresas a través de FUNDAE. Casi 340.000 empresas se beneficiaron de la bonificación por la formación realizada a sus empleados, de las que dos terceras partes fueron microempresas con menos de 9 trabajadores, y solamente el 1,2\% fueron grandes empresas con más de 249 trabajadores, siendo el resto pequeñas empresas (entre 10 y 49 trabajadores) o medianas empesas (de 50 a 249 trabajadores) (FUNDAE, 2020).

Curiosamente, del total de crédito asignado a las empresas para formación de sus empleados, dependiente de la cuota de formación profesional cotizada, y de la Ley de Presupuestos Generales del Estado, entre otros, las microempresas hacen uso del 71,5\% de su crédito, mientras que las grandes empresas llegan al $67,5 \%$. En relación a la formación superior, uno de los datos más interesantes de este informe, es que el $70,9 \%$ de los trabajadores que han disfrutado de un permiso para realizar cursos oficiales (Permisos Inidividuales de Formación, PIF) ha sido para obtener un título Universitario (FUNDAE, 2020), lo que pone de relevancia la importancia para las empresas de tener titulados superiores en su plantilla.

Así se ve reflejado también en los datos del Centro de Formación Permanente de la Universitat Politècnica de València (CFP), donde la formación para empresas representó en 2020 tres cuartas partes de la formación bajo demanda, siendo responsable del 36\% de los ingresos totales (CFP, 2020). La relevancia de la formación para trabajadores de empresas, también se deja ver en los alumnos inscritos en los cursos de posgrado ofrecidos por el Grupo de Ingeniería y Tecnología del Agua (ITA) perteneciente al Departamento de Ingeniería Hidráulica y Medio Ambiente de la Universitat Politècnica de València. De los 23 cursos individuales y 5 títulos propios que llevan impartiéndose como posgrado desde el curso 2016/2017, si se divide el perfil de los estudiantes entre los que provienen de empresa, y los que se inscriben de forma particular, más de la mitad de los estudiantes se inscriben a través de su empresa. Concretamente, en el 
último curso académico 19/20 esta cifra fue del 75\%, solicitando la bonificación a través de FUNDAE el $39,7 \%$ de estas empresas y siendo el $24,7 \%$ estudiantes de empresas extranjeras.

Entre toda la oferta formativa del ITA, destaca la realacionada con el software EPANET, un programa gratuito extensamente utilizado en el mercado laboral de la gestion de redes de agua a presión, ya sean de distribución o de riego, por lo que muchas de las empresas de este sector lo utilizan en su día a día. Estas empresas buscan una formación específica en el dominio de este software, inscribiendo de forma asidua a sus empleados en alguno de los 6 cursos relacionados con este programa, incluso en el título de posgrado Experto Universitario en EPANET que engloba los 6 módulos ofertados.

De los 2380 alumnos que han pasado por la formación a distancia ofertada por el ITA desde sus inicios, 885 han realizado alguno de los módulos relacionados con EPANET. De todos estos alumnos aproximadamente el $60 \%$ provienen de empresas, tanto nacionales como internacionales. Se cuenta con empresas de todo tipo, desde microempresas hasta grandes empresas, así como alumnos de la administración pública.

Concretamente, el curso Análisis de redes de agua con EPANET es el que mayor demanda tiene, comenzando a impartirse de manera presencial y pasando a modalidad online en 2010, formando parte a día de hoy como asignatura en 3 de las 5 titulaciones de posgrado a distancia ofertadas. Sin ir más lejos, en el curso 2019/2020, de entre los 23 cursos ofertados por el ITA, el de Análisis de redes de agua con EPANET fue el que mayor número de alumnos tuvo, representando el 12,6\% del total de estudiantes. En los últimos 6 años este curso ha tenido 339 alumnos, siguiendo la tendencia de ser el curso con más alumnos en las diferentes ediciones celebradas. De estos 339 alumnos, 178 se han inscrito por su cuenta y 161 provienen de empresa, dividiéndose éstos en 118 alumnos pertenecientes a grandes empresas y 43 de pequeñas empresas (Fig.1).

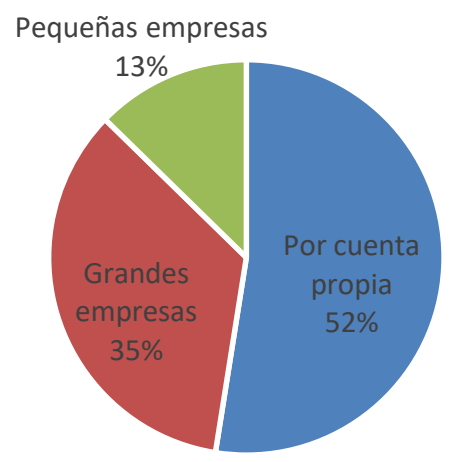

Fig. 1 Porcentaje de alumnos inscritos en el curso Análisis de redes de agua con EPANET en función de su procedencia

Los contenidos y la metodogía de formación es idéntica para los estudiantes que vienen de empresas, y para aquellos estudiantes que lo realizan por cuenta propia. En este trabajo se analiza en profundidad si los alumnos procedentes de empresas y los alumnos inscritos por cuenta propia cuentan con un comportamiento diferente. Para ello, se estudiarán las diferentes variables que afectan al desarrollo del curso y los resultados obtenidos por parte de ambos grupos de estudiantes. 


\section{Objetivos}

De los estudios e informes analizados en la introducción, se puede concluir que las empresas apuestan por contar en sus filas con empleados formados, manteniendo una formación continua mientras desarrollan sus labores en la empresa. Sin embargo, desde el equipo de tutorización se ha detectado que este grupo de alumnos, en general, obtiene peores resultados y su motivación es menor. El objetivo principal de este trabajo es detectar las diferencias más significativas entre los alumnos que provienen de empresa y los alumnos que se inscriben de forma individual al curso online Análisis de redes de agua con EPANET. Para ello se analizarán variables como el tiempo de dedicación, los resultados obtenidos o las interacciones entre alumnos y tutores. Esto permitirá concluir si las impresiones de los tutores son un hecho, y establecer un objetivo secundario como es averiguar la causa de la posible desmotivación del alumnado en función de si provienen de pequeña empresa, gran empresa o son alumnos independientes, tal como se verá en el punto 4.1 .

En función de los resultados obtenidos se tomarán una serie de medidas innovadoras para tratar de equiparar el proceso de aprendizaje y los resultados de ambos tipos de estudiantes. De esta manera, los tutores podrán actuar en consecuencia y adaptar el aprendizaje para conseguir una mayor implicación en el curso por parte de aquel grupo de estudiantes que tenga peores resultados en las variables analizadas.

\section{Desarrollo de la innovación}

\subsection{Descripción del curso objeto del análisis}

El análisis de este trabajo se va a realizar sobre el curso Análisis de redes de agua con EPANET. Este curso tiene una carga lectiva estimada de $60 \mathrm{~h}$, dividiéndose en 5 unidades que contemplan una serie de evaluaciones con el objetivo de garantizar que los conocimientos se van adquiriendo y consolidando por parte de los estudiantes (del Teso, et al. 2018).

La calificación final se obtiene a partir de 4 evaluaciones diferentes: puntos de control o "checkpoints", test de las unidades, test final del curso y entrega de ejercicios. Los puntos de control permiten verificar el grado de compresión del alumno sobre el contenido desarrollado hasta el momento, representado el $10 \%$ de la nota final. Al final de cada unidad, los estudiantes deberán responder un test en el que se preguntan los conceptos teóricos principales estudiados en la unidad, esta parte representa el 10\% de la nota final. Para reafirmar la comprensión de estos conceptos, una vez finalizados todos los contenidos, el alumno se enfrentará a un test final que reúne preguntas sobre todos los conceptos teóricos y prácticos que se han visto en el curso, representando esta parte de la evaluación el $40 \%$ de la nota final.

El curso de Análisis de redes de agua con EPANET es muy práctico, los alumnos deben trabajar continuamente con el programa para resolver los problemas y casos prácticos planteados. En la última unidad, se plantea la resolución de 5 ejercicios prácticos que deberán enviar resueltos para que los profesores los evalúen y proporcionen una retroalimentación acera de la idoneidad de la solución propuesta. Hasta que el ejercicio no está bien realizado no se da por válida su entrega. La evaluación de los ejercicios representa el $40 \%$ de la nota final del curso.

Con este sistema de evaluación se pretende lograr que el alumno adquiera los conocimientos mínimos exigidos para superar la formación (Bhuasiri et al., 2011).

\subsection{Descripción de la muestra}

La muestra cuenta con 339 observaciones recogidas desde la edición 15-16 hasta el presente curso académico 20-21. Dicha muestra está formada tanto por alumnos provenientes de empresas (161) como 
alumnos por cuenta propia (178). Para cada alumno se cuenta con los siguientes datos, que a priori se estima que pueden influir en los resultados obtenidos:

- Edición en la que realizaron la formación.

- El tipo de formación realizada, es decir, si han desarrollado los contenidos del curso al estudiar alguno de los 3 títulos de posgrado de los que forma parte la asignatura Análisis de redes de agua con EPANET o si se han inscrito únicamente en el curso.

- Si son alumnos inscritos por libre o han realizado la formación a través de empresa. En este último caso, también se recoge si se trata de una empresa de gran tamaño (más de 249 trabajadores siguiendo el criterio de FUNDAE) o pequeño tamaño (menos de 249 trabajadores).

- La nota obtenida en las evaluaciones de test, test final y ejercicios; la calificación final del curso y si lo han finalizado por completo o no.

- El tiempo en el que han visualizado los contenidos (dicho tiempo no contabiliza la realización de los ejercicios).

- Número de interacciones con los tutores a través del sistema de tickets interno de la plataforma interactiva en la que los alumnos desarrollan los contenidos.

\subsection{Análisis estadístico}

Los objetivos del estudio son, en primer lugar, analizar estadísticamente los datos para determinar si los resultados de los alumnos difieren en función de si provienen de empresa o se matriculan por libre, tal y como recogen las impresiones de los tutores.

Para ello, tras un análisis descriptivo de la muestra, se realizará un análisis de varianza (ANOVA) para dectectar si las notas medias de los alumnos varían en función del grupo al que pertenecen: alumno de empresa o alumno matriculado de forma particular. En el caso de que así sea, se analizarán en detalle las distintas características de ambos grupos para detectar la posible causa de dicha diferencia.

Para que el análisis ANOVA sea válido, las muestras deberán cumplir las siguientes condiciones:

1. Ser aleatorias: En este caso, este supuesto se cumple al tratarse cada observación de un alumno diferente.

2. Seguir una distribución normal.

3. Contar con una varianza similar.

En el caso en el que los datos no sigan una distribución normal, y/o las varianzas no sean semejantes, no serían válidos los resultados obtenidos a través del análisis ANOVA. Por lo tanto, se realizaría la prueba de Kruskal-Wallis. Dicha prueba, en lugar de analizar la diferencia entre las medias, evalúa la diferencia entre las medianas. Sin embargo, la prueba de Kruskal-Wallis determina si hay diferencia entre la mediana de los grupos, pero no entre cuáles. Así, en el caso de que haya más de dos grupos, el análisis se complementará con la prueba de la Mediana de Mood, que permitirá determinar entre qué grupos hay diferencias estadísticamente significativas. El análisis se ha realizado mediante el software Statgraphics Centurion XVI.

Si efectivamente resulta que ambas muestras son distintas, se valorará la motivación e implicación de los alumnos de ambos grupos como posibles causas de dicha diferencia. Para ello se estudiará el tiempo dedicado a la visualización de los contenidos, ya que los tutores tienen la impresión que los alumnos de empresa, en general, realizan el curso en menos horas y prestándole menos atención. 
Otra de las variables a analizar que es de interés para concluir la implicación de los alumnos en el curso, es la cantidad de consultas realizadas a los tutores. El sistema de consultas puede realizarse a través de correo electrónico o a través de la plataforma educativa en la que se desarrolla el curso, la cual permite el envío de consultas a través de los denominados "tickets". De forma cualitativa, los tutores han percibido menos participación de los alumnos provenientes de empresas, más aún si son de grandes entidades.

Además de la falta de interacción con el profesorado, otro de los problemas detectados, que son específicos de los alumnos de grandes empresas, son el plagio en algunos ejercicios e informes enviados. Situaciones como que la fecha del ejercicio enviado sea previa a que el estudiante haya llegado al punto del curso en el que se explica el ejercicio, o el envío de ejercicios resueltos perfectamente por alumnos con bajas calificaciones en los puntos de control y con una dedicación de unas pocas horas, hacen pensar que los ejercicios enviados no los ha realizado el propio estudiante.

\section{Resultados}

Tras un primer análisis descriptivo realizado sobre la muestra de las notas finales alcanzadas por los alumnos en función del grupo al que pertenezcan: alumno de empresa o alumno matriculado de forma particular, se observa en la tabla 1, que los elevados valores de curtosis estandarizada denotan que la muestra no sigue una distribución normal. Por tanto, no se puede realizar el análisis ANOVA para determinar si existe diferencia entre ambos grupos.

Tabla 1. Resultados del análisis descriptivo de la nota final en función del tipo de alumno

\begin{tabular}{|l|c|c|l|c|c|c|c|c|c|}
\hline $\begin{array}{l}\text { Tipo de } \\
\text { alumno }\end{array}$ & Recuento & Promedio & $\begin{array}{l}\text { Desviación } \\
\text { Estándar }\end{array}$ & $\begin{array}{l}\text { Coeficiente } \\
\text { Variación }\end{array}$ & Mínimo & Máximo & Rango & $\begin{array}{l}\text { Sesgo } \\
\text { Estand. }\end{array}$ & $\begin{array}{l}\text { Curtosis } \\
\text { Estand. }\end{array}$ \\
\hline No empresa & 178 & 8,33 & 2,46 & $29,5 \%$ & 0 & 9,9 & 9,9 & $-13,56$ & 13,64 \\
\hline Empresa & 161 & 7,95 & 2,48 & $31,23 \%$ & 0,2 & 9,9 & 9,7 & $-10,65$ & 8,09 \\
\hline Total & 339 & 8,15 & 2,47 & $30,33 \%$ & 0 & 9,9 & 9,9 & $-16,93$ & 14,70 \\
\hline
\end{tabular}

Por esta razón, se emplea la prueba de Kruskal-Wallis para determinar si las medianas de la muestra difieren. Dicho análisis concluye que existe una diferencia significativa entre ambos grupos, con un $95 \%$ de confianza, confirmándose que los alumnos pertenecientes a empresa obtienen peores resultados, siendo la mediana 1.3 puntos inferior para este grupo.

Sin embargo, el grupo de alumnos que provienen de empresa es muy diverso, contando por un lado con aquellos provenientes de grandes corporaciones y, por el otro, los que forman parte de pequeñas empresas. Es por ello que se ha replicado el análisis anterior considerando dicho factor, quedando la muestra dividida en: alumnos de empresa grande (118), alumnos de empresa pequeña (43) y alumnos que no pertenecen a empresa (178). Tal y como se observa en la tabla 2 , los resultados obtenidos para alumnos pertenecientes a pequeñas corporaciones son más similares a aquellos alumnos inscritos de forma particular, obteniendo incluso mejores resultados que éstos de media.

Tabla 2. Resultados del análisis descriptivo de la nota final en función del tipo de alumno y el tamaño de empresa

\begin{tabular}{|l|c|c|c|l|c|c|c|c|c|}
\hline $\begin{array}{l}\text { Tipo de } \\
\text { alumno }\end{array}$ & Recuento & Promedio & $\begin{array}{l}\text { Desviación } \\
\text { Estándar }\end{array}$ & $\begin{array}{l}\text { Coeficiente de } \\
\text { Variación }\end{array}$ & Mínimo & Máximo & Rango & $\begin{array}{l}\text { Sesgo } \\
\text { Estand. }\end{array}$ & $\begin{array}{l}\text { Curtosis } \\
\text { Estand. }\end{array}$ \\
\hline E. Grande & 118 & 7,55 & 2,66 & $35,28 \%$ & 0,3 & 9,9 & 9,6 & $-7,40$ & 3,41 \\
\hline No empresa & 178 & 8,33 & 2,46 & $29,47 \%$ & 0 & 9,9 & 9,9 & $-13,56$ & 13,64 \\
\hline
\end{tabular}




\begin{tabular}{|l|c|c|c|c|c|c|c|c|c|}
\hline E. pequeña & 43 & 9,02 & 1,44 & $15,92 \%$ & 0,2 & 9,8 & 9,6 & $-15,43$ & 48,05 \\
\hline Total & 339 & 8,15 & 2,47 & $30,33 \%$ & 0 & 9,9 & 9,9 & $-16,93$ & 14,70 \\
\hline
\end{tabular}

Como en el caso anterior, los elevados valores de curtosis imposibilitan analizar si dichas diferencias son significativas con un ANOVA. La prueba de Kruskal-Wallis determina que en este caso también existe una diferencia significativa entre grupos con un $95 \%$ de confianza. Sin embargo, no determina entre qué grupos se produce dicha diferencia. Es por ello que se lleva a cabo la prueba de la mediana de Mood (Tabla 3). Dicho análisis determina que el grupo de empresa grande es el que cuenta con unas calificaciones finales significativamente más bajas respecto a los otros dos grupos, mientras que los resultados de los alumnos de pequeñas empresas y los incritos de forma particular no son estadísticamente distintos. La Fig.2 muestra el diagrama de caja y bigotes que corrobora el análisis estadístico realizado.

Tabla 3. Resultados de la prueba de la Mediana de Mood para determinar la influencia del tipo de alumno y el tamaño de empresa en la nota final

\begin{tabular}{|c|c|c|c|c|c|c|}
\hline Tamaño empresa & Tamaño de Muestra & $\mathrm{n}<=$ & $\mathrm{n}>$ & Mediana & LC inferior 95,0\% & LC superior 95,0\% \\
\hline E. Grande & 118 & 83 & 35 & 8,6 & 8,3 & 8,71 \\
\hline No empresa & 178 & 74 & 104 & 9,2 & 9,1 & 9,3 \\
\hline E. pequeña & 43 & 15 & 28 & 9,3 & 9,0 & 9,5 \\
\hline
\end{tabular}

LC inferior y LC superior representan los intervalos de $95 \%$ de confianza, inferior y superior respectivamente, para la mediana. Siendo n el número de muestras que hay cada lado de la mediana.

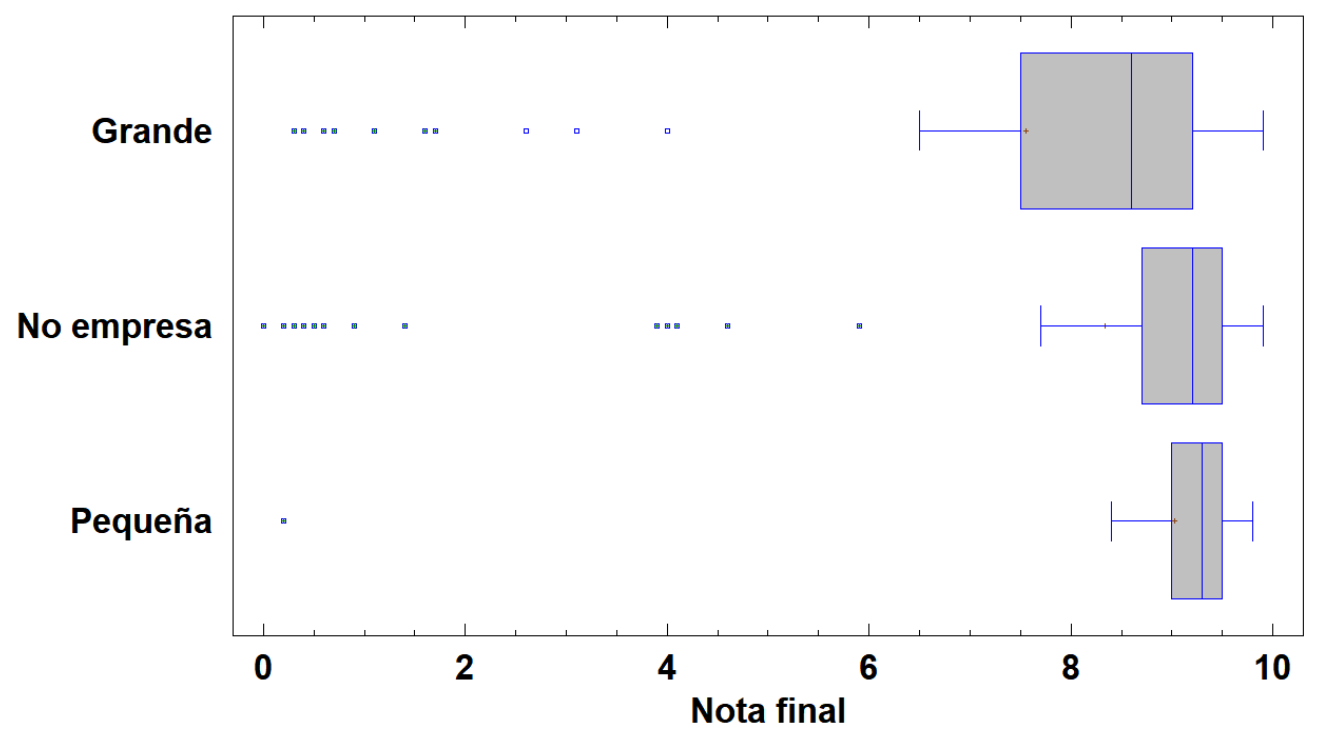

Fig. 2. Diagrama de caja y bigotes de la distribución de la nota final en función del tipo de alumno y el tamaño de la empresa

Además de que se ha demostrado que las calificaciones obtenidas por los alumnos de empresa grande son más bajas, un análisis en la tasa de abandono del curso corrobora esta diferencia, ya que el $18 \%$ de los alumnos de empresa grande no finalizan la formación, frente al $11 \%$ de alumnos particulares y el $2 \%$ de alumnos de empresa pequeña que, con diferencia, es el grupo con mayor tasa de éxito.

Una vez confirmado que existe una diferencia entre los resultados alcanzados por alumnos de empresa grande y el resto de alumnos, se van a a anlizar las posibles causas que llevan a esta situación. 


\subsection{Causas de la diferencia de resultados}

Una de las posibles causas del peor desempeño por parte de los alumnos de empresa grande es su motivación para realizar el curso y su implicación en éste. Para ello, en primer lugar se analizará el tiempo dedicado a la visualización de los contenidos para los tres grupos analizados (empresa grande, empresa pequeña y alumno particular) y si éste tiene un impacto en la nota final obtenida. Para ello, se clasifica el tiempo de visualización de contenidos en 3 categorías:

- Menos de $15 \mathrm{~h}$.

- Entre 15 y $30 \mathrm{~h}$.

- Más de 30h.

Cabe recordar que se trata de una formación de $60 \mathrm{~h}$ en las que el tiempo de visualización contabiliza el número de horas que el alumno emplea en visitar los contenidos, pero dicho tiempo no contempla la realización de los ejercicios prácticos.

Un análisis del tiempo empleado para la visualización de los contenidos por tipo de alumno (Fig.3) denota que los alumnos provenientes de empresas grandes son los que menos tiempo dedican a la visualización de contenidos, siendo el grupo dominante en la categoría de tiempo "Menos de $15 \mathrm{~h}$ " y el que menos representación tiene en la categoría de "Más de 30h". Los alumnos de pequeñas empresas, en cambio, muestran un comportamiento completamente distinto y más semejante al de los alumnos inscritos por cuenta propia, con la salvedad de que son el grupo de alumnos que más tiempo dedica a la visualización de contenidos.

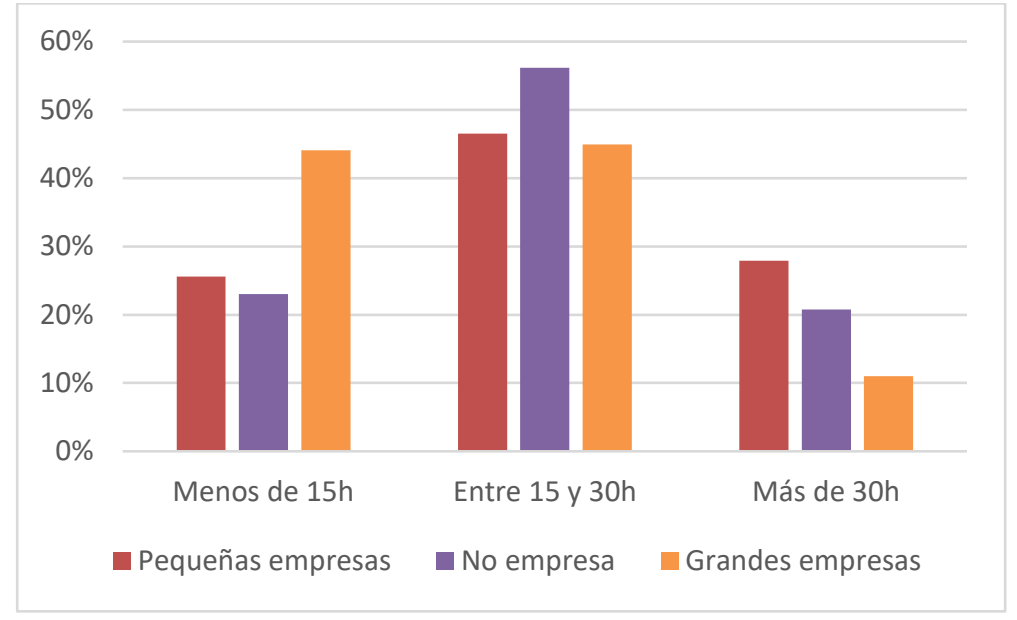

Fig. 3 Tiempo en el que los alumnos visualizan los contenidos por tipo de alumno

Para deteminar si dicha diferencia en la visualización de contenidos es uno de los factores que influye en los resultados más bajos obtenidos por los alumnos de empresa grande, se analiza si dicho factor cuenta con un impacto estadísticamente significativo en la nota final.

Al tratarse de una muestra que no sigue una distribución normal, se realiza la prueba de Kruskal-Wallis, que confirma que sí que hay diferencia estadística entre los grupos, con un nivel de confianza del 95\%. La realización posterior de la Prueba de la Mediana de Mood confirma que, concretamente, dicha diferencia se encuentra en el grupo de alumnos que realizan la formación en menos de $15 \mathrm{~h}$, tal y como muestra la tabla 4, siendo la mediana de este grupo 0.5 puntos inferior a la de la categoría de "Entre 15 y 30 h". 
Tabla 4. Resultados de la prueba de la Mediana de Mood para analizar la influencia del factor tiempo en los resultados finales

\begin{tabular}{|l|c|c|c|c|c|c|}
\hline Tiempo & Tamaño de Muestra & $\mathrm{n}<=$ & $\mathrm{n}>$ & Mediana & LC inferior 95,0\% & LC superior 95,0\% \\
\hline Menos de 15h & 107 & 76 & 31 & 8,6 & 8,3 & 8,813 \\
\hline Entre 15 y 30h & 173 & 77 & 96 & 9,1 & 9,0 & 9,3 \\
\hline Más de 30h & 59 & 19 & 40 & 9,3 & 9,1 & 9,4 \\
\hline
\end{tabular}

LC inferior y LC superior representan los intervalos de $95 \%$ de confianza, inferior y superior respectivamente para la mediana. Siendo n el número de muestras que hay cada lado de la mediana.

El gráfico de caja y bigotes de la nota final en función del tiempo de visualización de los contenidos (Fig 4), confirma que mientras que el grupo de "menos de $15 \mathrm{~h}$ " tiene una distribución muy amplia, hecho que demuestra el tamaño de la caja, los grupos de "entre 15 y 30h" y "más de 30h" tienen un comportamiento más uniforme y similar entre ellos.

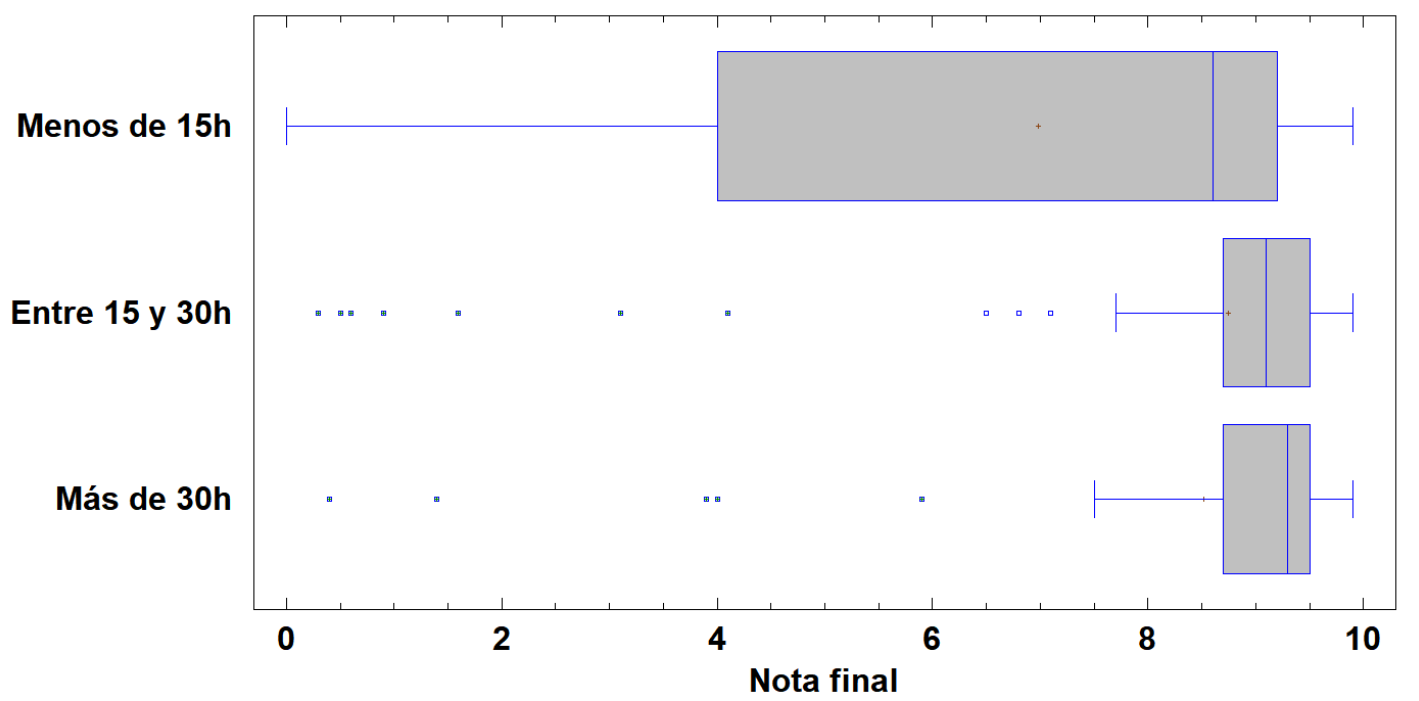

Fig. 4 Gráfico de caja y bigotes de la nota final en función del tiempo en el que se visualizan los contenidos

Otra forma de valorar la motivación e implicación del alumno es analizando el número de interacciones que realiza con los tutores. Para ello, se ha analizado el número de consultas efectuadas por los alumnos a través del sistema de tickets de la plataforma de formación a distancia en la que se desarrollan los contenidos.

El análisis se ha realizado únicamente para el grupo de alumnos inscritos en el curso individual, ya que los alumnos de título propio, al cursar más asignaturas, cuentan con una mayor interacción.

El análisis confirma que únicamente el $21 \%$ de los alumnos de empresa grande han contactado con los tutores a través del sistema de tickets, siendo el promedio de consultas de 1.8 tickets por alumno. Los alumnos de empresa pequeña cuentan con una mayor interacción con los tutores, ya que el $44 \%$ de ellos ha contactado en algún momento a través del sistema tickets, con una media de 2.89 tickets por alumno. Dicho comportamiento es similar al de los alumnos inscritos de forma particular, con un $38 \%$ que ha contactado con los tutores y una media de 2.88 tickets por alumno. Esta diferencia de comportamiento entre los distintos grupos de alumnos es identificable a través del gráfico de caja y bigotes que representa el número de tickets escritos por los diferentes tipos de alumnos (Fig. 5). 


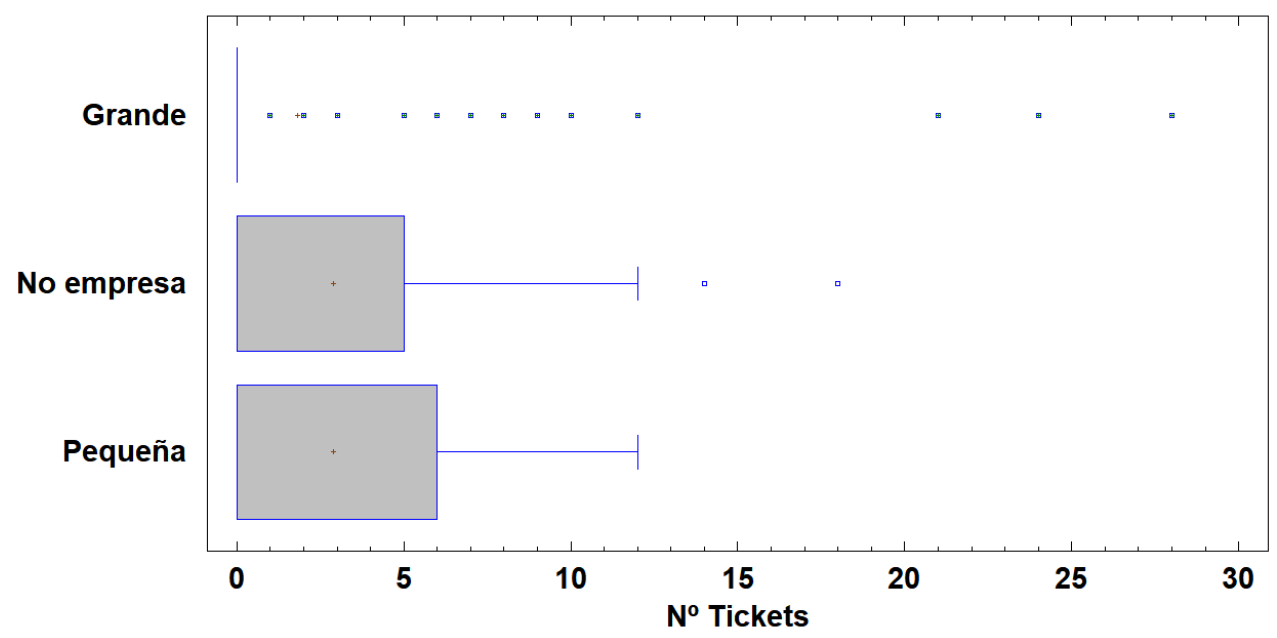

Fig. 5 Gráfico de caja y bigotes del número de tickets para los distintos tipos de alumnos de cursos

\subsection{Impacto del seguimiento del curso en los resultados obtenidos}

El seguimiento del aprendizaje a distancia es una herramienta clave para mantener al alumno motivado, pero también para informale de cómo está realizando el curso y que perciba que, a pesar de tratase de formación online, cuenta con tutores detrás de la pantalla brindándole apoyo.

El número de alumnos y de cursos de la formación Cursosagua ha ido incrementándose a lo largo del tiempo, contando actualmente con 23 cursos, 5 títulos online de posgrado y entre 200 y 300 alumnos inscritos en los distintos cursos y títulos anualmente. Con tal cantidad de alumnos, el seguimiento manual de cada uno de ellos se hace inviable para los tutores.

Hasta el curso académico 15-16 el seguimiento de dichos alumnos se realizaba de forma completamente manual. En la edición 16-17, coincidiendo con el lanzamiento del Plan de Estudios Coordinado, se implementó un seguimiento semi-manual realizado tres veces al año. Finalmente, en el pasado curso académico se implementó una herramienta semiautomática de seguimiento para facilitar a los tutores las tareas de seguimiento y permitir un seguimiento más frecuente (Estruch-Juan et al., 2020).

Así, se ha analizado si las mejoras en el seguimiento han tenido efectivamente un incremento en los resultados obtenidos por los alumnos. Tal y como muestra la tabla 5 y en la Fig.6, el promedio y la mediana de los alumnos da un salto importante a partir del curso 16-17 y otro en el presente curso académico. Dichas mejoras coinciden en el tiempo con las mejoras llevadas a cabo en el seguimiento de los alumnos, con la única excepción del curso académico 19-20, cuyos resultados se mantuvieron relativamente constantes sin grandes cambios a pesar de las mejoras en el seguimiento. Dicho resultado es esperable debido a la situación sanitaria experimentada a lo largo del curso.

Tabla 5. Evolución de las notas finales a lo largo de las ediciones

\begin{tabular}{|l|c|c|c|}
\hline Edición & Recuento & Promedio & Mediana \\
\hline $15-16$ & 74 & 6,95 & 8,2 \\
\hline $16-17$ & 60 & 8,27 & 9 \\
\hline $17-18$ & 63 & 8,22 & 9,3 \\
\hline $18-19$ & 41 & 8,70 & 9,3 \\
\hline $19-20$ & 63 & 8,57 & 9,3 \\
\hline $20-21$ & 38 & 8,95 & 9,4 \\
\hline
\end{tabular}




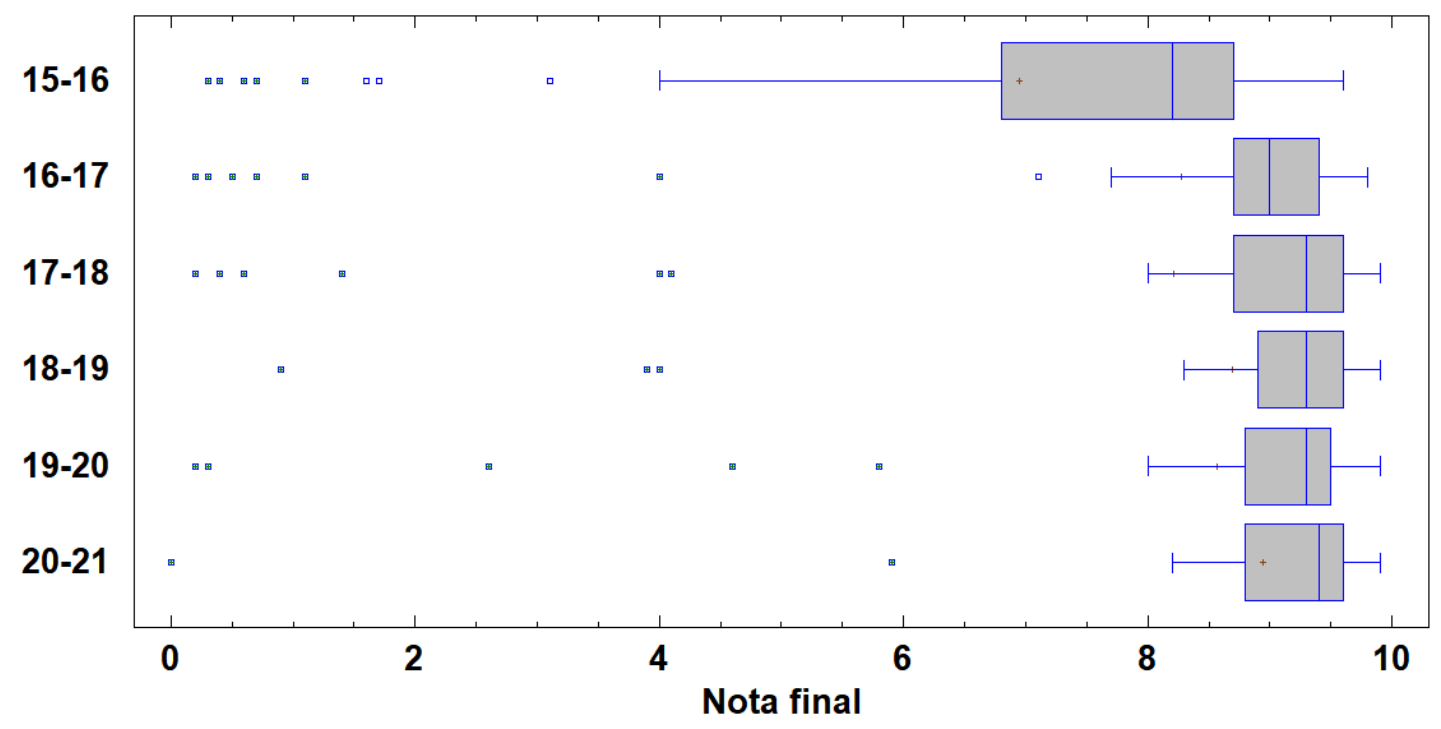

Fig.6. Gráfico de caja y bigotes para la evolución de los resultados a lo largo de las ediciones

\section{Conclusiones}

Tras evaluar los resultados del análisis estadístico realizado, se concluye que los alumnos provenientes de grandes empresas obtienen peores calificaciones e interactuan menos con los tutores, lo que denota una falta de motivación por realizar el curso. Un posible motivo es la formación masiva que reciben los trabajadores de estas grandes corporaciones, inscribiendo habitualmente a un elevado número de alumnos, sin ser quizá la formación que más les interese recibir, y viéndose en la obligación de tener que realizarla de manera forzosa. De manera anecdótica, se ha llegado a dar el caso en el que una gran empresa ha inscrito a un mismo alumno en el curso dos veces, lo que denota la posible falta de planificación en la formación de este tipo de empresas, derivándose en alumnos con menos motivación, menos dedicación al curso y por ende, peores resultados.

En el lado opuesto, se cuenta con alumnos de pequeñas empresas, los cuales obtienen una mejor calificación, siendo el grupo con más representatividad de los que dedican más de $30 \mathrm{~h}$ a la formación. Además, estos estudiantes son los que mayor tasa de éxito tienen, siendo únicamente el $2 \%$ los que no finalizan, frente al $18 \%$ que representan las grandes empresas.

En la mayoría de ocasiones, en este tipo de estudiantes, son ellos mismos los que se interesan por la formación, contactando ellos directamente y haciendo las gestiones pertinentes para poder bonificar el curso a través de su empresa. Son conscientes de sus necesidades en el puesto que ocupan, y buscan una formación específica para mejorar en el mismo, lo que se traduce en una mayor motivación y mejores resultados.

En definitiva, el estudio llevado a cabo ha permitido detectar una serie de diferencias entre alumnos que no son de empresa, los que provienene de pequeñas empresas y los pertenecientes a grandes entidades. Con el objetivo de solventar la problemática detectada en alumnos de grandes empresas, y conocida la mejora que supuso la implementación hace unos años del sistema de seguimiento que se lleva a cabo actualmente por parte de los tutores, se planifica realizar en las ediciones futuras de la formación del ITA seguimientos más habituales para alumnos de grandes corporaciones. La intención es hacerles conocer de manera específica y personal cual es su avance y resultado en las diferentes etapas del curso, tratando de alentar al estudiante a continuar con el curso y no dejarlo para el final. 
Por otro lado, se plantea readaptar el peso de las evaluaciones, ya que actualmente el Test Final representa un $40 \%$ de la nota final, y quizá sea más conveniente dar un mayor peso a los checkpoints, ya que son el tipo de evaluación que permite calificar de forma más afín la evaluación contínua de los alumnos. Incrementando el peso de los checkpoints en la nota final, se espera que los alumnos presten una mayor atención a esta evaluación, volviendo a visualizar y repasar los contenidos con mayor profundidad en el caso de no superar estos puntos de control.

Por último, con la intención de dar a conocer al alumno el sistema de calificación seguido por parte de los tutores, se creará una rubrica de evaluación para los ejercicios, lo que orientará a los alumnos en cómo enfocar su resolución y dónde deben hacer un mayor enfásis para obtener una buena calificación.

\section{Referencias}

BHUASIRI W., XAYMOUNGKHOUN O., ZO H., JAE JEUNG RHO J., CIGANEK A. (2011). "Critical success factors for e-learning in developing countries: A comparative analysis between ICT experts and faculty" Computers \& Education 58(2):843-855 (February 2012) DOI10.1016/j.compedu.2011.10.010

CENTRO DE FORMACIÓN PERMANENTE DE LA UNIVERSITAT POLITÈCNICA DE VALÈNCIA (2020). Memoria de actividades 2019-2020. Valencia: Universitat Politècnica de València.

CRUE (2018). La universidad española en cifras 2017-2018. CRUE Universidades Españolas.

DEL TESO, R., ESTRUCH, E., GÓMEZ, E., SORIANO, J. (2018). "Sistema de evaluación para la formación a distancia de profesionales" en Congreso Nacional de Innovación Educativa y de Docencia en Red. IN-RED 2018

ESTRUCH-JUAN, E., DEL TESO, R., GÓMEZ, E., SORIANO, J. (2020). "Herramienta para el seguimiento del aprendizaje a distancia en alumnos de posgrado. El potencial de Office para realizar envíos personalizados" en Congreso Nacional de Innovación Educativa y de Docencia en Red. IN-RED 2020

FUNDACIÓN ESTATAL PARA LA FORMACIÓN EN EL EMPLEO. FUNDAE (2020). Formación para el empleo. Balance de situación 2019. FUNDAE.

INSTITUTO NACIONAL DE ESTADÍSTICA. INE (2020). Encuesta de inserción laboral de titulados universitarios. EILU. Año 2019. INE.

OLIVER, J. (2018). El Futuro del Empleo. El impacto de la transformación productiva y la revolución demográfica en la creación de empleo 2017-2026. ManpowerGroup.- 